\title{
Evidence of low vitamin D intake and suboptimal 25-hydroxyvitamin D status in adolescent females: data from a 3-year longitudinal study on bone health
}

\author{
J. A. Nurmi-Lawton ${ }^{1}$, A. Baxter-Jones ${ }^{2}$, P. Taylor ${ }^{3}$, C. Cooper ${ }^{3}$, J. L. Berry ${ }^{4}$ and S. A. Lanham-New ${ }^{5}$ \\ ${ }^{1}$ Helsinki University Central Hospital, Helsinki, Finland, ${ }^{2}$ University of Saskatchewan, Saskatoon, Saskatchewan, Canada, \\ ${ }^{3}$ University of Southampton, Southampton SO16 6YD, UK, ${ }^{4}$ Vitamin D Research Group, University of Manchester, \\ Manchester M13 9WL, UK and ${ }^{5}$ Nutritional Sciences Division, Faculty of Health and Medical Sciences, \\ University of Surrey, Guildford GU2 7XH, UK
}

Vitamin D deficiency has been associated adversely with health outcomes. There is no dietary reference value for vitamin D for the agegroup 4-64 years as it is considered that UVB exposure from sunlight provides sufficient quantities. There is now overwhelming evidence of widespread vitamin D insufficiency in the general population. The optimum levels of serum 25-hydroxyvitamin D (S-25(OH)D) for adult health are considered to be $75-80 \mathrm{nmol} / \mathrm{l}$, but the levels required for children and adolescents to maintain optimal peak bone mass $(\mathrm{PBM})$ are not clear $^{(1)}$.

The effects of nutrition and exercise on PBM development were investigated in young female gymnasts $(\mathrm{G})$ and non-gymnasts $(\mathrm{C})^{(2)}$. The initial baseline data for this 3-year longitudinal study are reported here. Dietary intake was assessed using estimated dietary records (7 d at baseline) and analysed using Diet 5 for Windows (version 2000; Robert Gordon University, Aberdeen, UK). Blood samples were collected during the month of October and S-25(OH)D, plasma parathyroid hormone (P-PTH), serum Ca (S-Ca) and serum albumin (S-alb) were determined at baseline. Data for anthropometric measurements, pubertal maturation, bone mass and bone metabolism markers were also collected and reported previously ${ }^{(1)}$.

\begin{tabular}{|c|c|c|c|c|}
\hline & \multicolumn{2}{|c|}{$\mathrm{G}(n 38)$} & \multicolumn{2}{|c|}{$\mathrm{C}(n 46)$} \\
\hline & Mean & SD & Mean & SD \\
\hline Age (years) & 11.2 & 2.2 & 11.5 & 1.8 \\
\hline Height $(\mathrm{m})$ & $1.36^{\mathrm{a}}$ & 0.1 & $1.49^{\mathrm{b}}$ & 0.1 \\
\hline Weight $(\mathrm{kg})$ & $31.1^{\mathrm{a}}$ & 7.9 & $41.7^{\mathrm{b}}$ & 11.4 \\
\hline Vitamin D intake $(\mu \mathrm{g} / \mathrm{d})$ & 2.6 & 1.8 & 2.4 & 1.3 \\
\hline $\mathrm{S}-25(\mathrm{OH}) \mathrm{D}(\mathrm{nmol} / \mathrm{l})$ & $46.6^{\mathrm{a}}$ & 16.0 & $55.9^{\mathrm{b}}$ & 17.2 \\
\hline P-PTH $(\mathrm{pmol} / \mathrm{l})$ & 2.3 & 1.0 & 2.1 & 0.9 \\
\hline $\mathrm{S}-\mathrm{Ca}(\mathrm{mmol} / \mathrm{l})$ & $2.2^{\mathrm{a}}$ & 0.06 & $2.3^{\mathrm{b}}$ & 0.05 \\
\hline S-alb $(g / l)$ & $46.4^{\mathrm{a}}$ & 1.7 & $45.3^{\mathrm{b}}$ & 1.8 \\
\hline
\end{tabular}

${ }^{\mathrm{a}, \mathrm{b}}$ Means within rows with unlike superscript letters were significantly different $(t$ test, $P<0.05)$.

Dietary intakes of vitamin D and Ca were similar for both groups. Group G had significantly lower S-25(OH)D and S-Ca (all $P<0.01$ ) than group C, but no difference was found for P-PTH. S-25(OH)D levels $<40 \mathrm{nmol} / 1$ (indicative of suboptimal vitamin D status) was found in $34 \%$ of group $\mathrm{G}$ and $20 \%$ of group $\mathrm{C}$. There was a weak but significant Pearson correlation between dietary intake of vitamin D and S-25(OH)D for all subjects $(r 0.3, P<0.02)$ and for group $\mathrm{G}(r 0.5, P<0.01)$, but not for the group C. A negative correlation was found between S-25(OH)D and P-PTH $(r-0.3, P<0.02)$.

These results indicate a prevalence of suboptimal vitamin D status in young British females. Group $\mathrm{G}$ have previously been reported to have greater bone mass than group $\mathrm{C}^{(1)}$. The finding of lower S-25(OH)D for group G but similar P-PTH compared with group C suggests that high-impact training may have independent bone-building effects through force loading, which may override other negative effects such as low S-25(OH)D status, thus allowing for optimal PBM development.

This work was funded by the National Osteoporosis Society. 